\title{
CLINICAL EVALUATION OF INTRAMASSETERIC VERSUS SUBMUCOSAL DEXAMETHASONE ADMINISTRATION AFTER SURGICAL REMOVAL OF IMPACTED MANDIBULAR THIRD MOLAR
}

\author{
Eslam A Mohamed*, Abdul Mnoiem A Ghaffar**
}

\begin{abstract}
Objective: The study was designed to compare the efficacy of intramassetric and submucosal routes of dexamethasone administration after surgical removal of impacted mandibular third molar. Materials and methods: This study was conducted on 30 patients of both sexes from the out-patient Clinics of oral and Maxillofacial surgery Department, Faculty of Dental Medecine, Boys, Cairo Al-Azhar University, and Sayed Jalal University Hospital. All patients has a mesio-angular, class II molar ( according to Pell and Gregory classification). The patients were divided randomly into three groups (10 patients each) crossly matched for age and sex. All the patients were subjected to complete history taking including name, age, sex, occupation, residence, chief complaint, medical and dental history. The patients were subjected to intraoral examination to determine the condition of soft tissue covering the impacted third molar, and the condition of pericoronal soft tissue. Panoramic views and a digital periapical X-rays films were taken for all patients to evaluate and classify the impacted third molars and amount of the bone around it. Assessment of pain level was done using visual analogue scale, Measurement of maximum mouth opening and edema. Results: In the $1^{\text {st }}, 3^{\text {rd }}$ and $7^{\text {th }}$ there was statistically significant difference between pain scores, mouth opening and edema in the two study groups and the control group. Conclusion: Pain reduction was noted in both study groups, Maximal mouth opening and edema improved with intramasseteric and submucosal groups more than in control group.
\end{abstract}

\section{INTRODUCTION}

The surgical extraction of impacted third molars is the most frequent minor surgical intervention in oral surgery ${ }^{(1,2)}$. This invasive procedure elicits an inflammatory response which may manifest mainly as pain, swelling, trismus. Sometimes, an exacerbated response may lead to moderate to severe short-term transient effects on the quality of life ${ }^{(3)}$. These post-extraction morbidities often become the reason for reluctance and hesitation in getting the tooth removed. Reduction of these comorbidities using several strategies has been an area of interest in the field of minor oral surgery. Consequently, a lot of research has gone into the field of pharmacological agents to reduce postextraction sequelae ${ }^{(4)}$.
Of all other teeth, wisdom teeth are the ones with the highest rate of remaining impacted. The extraction of impacted lower third molar teeth is one of the most common oral surgical procedures $(1,2)$. Often, complications which cause discomfort for patients occur with these operations ${ }^{(3)}$. Wisdom teeth that remain impacted in the jaw can cause complications such as pericoronitis, root resorption, tooth decay, periodontitis, infections (local and facial), cysts, tumors, and mandibular fractures ${ }^{(3)}$.

Complications such as alveolitis, infection, pain, swelling, trismus, hemorrhage, nerve damage, mandibular/tuberosity fracture, escape of the tooth or the root of the tooth to an anatomical site, adjacent tooth damage, or temporomandibular joint damage may occur after the surgery of impacted wisdom teeth ${ }^{(5)}$.

\footnotetext{
* Dentist, Ministry of Health.

** Professor, Department of Oral and Maxillofacial Surgery, Faculty of Dental Medicine, Boys, Cairo Al-Azhar University.
} 
Several protocols for the administration of dexamethasone in the third molar surgery have been proposed. Route of administration of dexamethasone has been a topic of contention with researchers still unable to find a consensus on the most effective way to reduce post-extraction sequelae ${ }^{(6-9)}$.

\section{AIM OF THE STUDY}

The aim of the present study was to compare the possible efficacy of intramassetric and submucosal routes of dexamethasone administration after surgical removal of impacted mandibular third molar.

\section{PATIENT AND METHODS}

All patients have a mesio-angular, class II molar (according to Pell and Gregory classification). The patients were divided randomly into three groups (10 patients each).

1. The control group

Patients in this group were not receive any form of corticosteroid.

2 . The study groups

Group A: $8 \mathrm{mg}$ dexamethasone was injected after the surgery into the intramasseteric (IM) muscle

Group B: $8 \mathrm{mg}$ dexamethasone was injected after the surgery around the operated site submucosal (SM).

\section{A -Preoperative phase}

\section{Clinical examination:}

All the patients were subjected to complete history taking including name, age, sex, occupation, residence, chief complaint, medical and dental history. The patients were subjected to intraoral examination to determine the condition of soft tissue covering the impacted third molar, and the condition of pericoronal soft tissue.

\section{Radiographic examination}

Panoramic views and a digital periapical X-rays films were taken for all patients to evaluate and classify the impacted third molars and amount of the bone around it.

\section{Measurements:}

1. Inter-incisal distance was measured in each patient by digital caliper which has two arms, one of them touch the mesio-incisal angle of upper incisors and the other touch the mesioincisal angle of the lower incisors.

2. The line between the tragus to the corner of the mouth was measured in each patient by flexible meters.

3. The line between the tragus to the pogonion was measured in each patient by flexible meters.

\section{B -Operative phase}

\section{Surgical procedure:}

- All the operations were performed under local anesthesia in Oral and maxillofacial Surgery Department, Al-Azhar University, Boys Cairo.

- Disinfection of the operation field was achieved with Betadine.

The mucoperiosteal flap was done by an incision with blade No 15, mounted on B.P scalpel handle No 3, starting just lingual to the external ridge of the ramus of the mandible. Then, the incision was continued bucally around the neck of the second molar to the interproximal space between the first and second molars.

This incision extend obliquely down toward the mucobuccal fold at a 45 degrees angle. The flap was the gently reflected with a periosteal elevator and gently retracted by Minnesota. The underlying bone over the impacted teeth was removed with a No 703 fissure surgical bur mounted on a straight angle low speed handpiece. Bone guttering was performed 
distal to the second molar from the mesio-buccal to disto-buccal aspects of the impacted teeth. The interlocked mesial cusps were removed by a fissure surgical bur mounted on high speed handpiece which splitting from buccal surface to lingual surface of the cusp. A suitable elevator was placed mesially at the neck of the tooth and rotated to move it distally and occlusally. Post-extraction, the socket was copiously irrigated using 5\% povidone-iodine solution diluted with equal parts of normal saline. The flap was sutured back with 3-0 silk sutures (BBS) using four interrupted sutures.

\section{The control group}

Patients in this group were not receive any form of corticosteroid.

\section{The study groups}

Group A: $8 \mathrm{mg}$ dexamethasone was injected after the surgery into the intramasseteric (IM) muscle. In group A, $8 \mathrm{mg}$ of phosphate disodium dexamethasone was administered to the masseter muscle. The technique consisted of a 2-mL injection containing $8 \mathrm{mg}$ of phosphate disodium dexamethasone injected into three different points. For the procedure, a 3-mL Luer-tip syringe was used, with a 22-gauge needle. The $2-\mathrm{mL}$ volume was then divided into equal thirds. The first third was injected with the needle parallel to the occlusal plane of the molars adjacent to the lower third molar to be extracted, directly reaching the mid-portion of the masseter. The second third was injected close to the mandible angle with the syringe forming a $45^{\circ}$ angle with the occlusal surface of the teeth of the same side. The last third was injected with the syringe forming a $90^{\circ}$ angle with the molars on the same side. The technique was performed as described by Messer and Keller ${ }^{[9]}$.

Group B: $8 \mathrm{mg}$ dexamethasone was injected after the surgery around the operated site submucosal (SM). In group B, $8 \mathrm{mg}$ of phosphate disodium dexamethasone was injected into the buccal vestibule near site of operation.

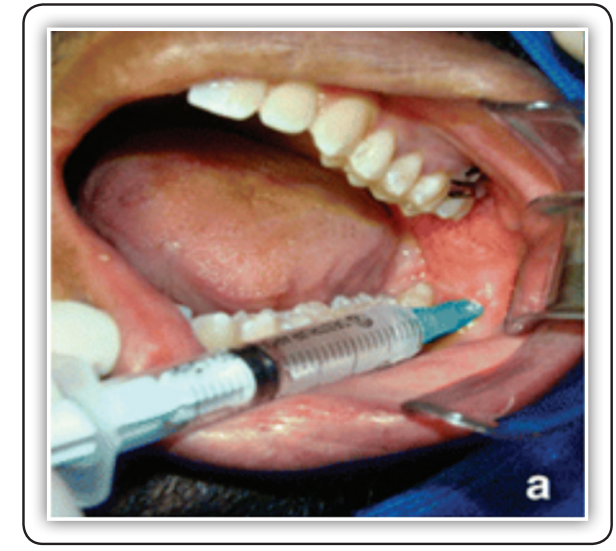

FIG (A) Photograph showing intramasseteric dexamethasone injection

\section{C-Postoperative phase}

\section{Postoperative evaluation was done clinically as follows:}

\section{Postoperative pain:}

Pain was evaluated at first, third, seventh postoperatively through the visual analogue scale (VAS) recorded from 0 to 10. (Appendix II) $0=$ "no pain" to $10=$ "the worse possible pain.

\section{Post-operative edema:}

Swelling was evaluated while the patient was sitting in an upright position. The measurements were taken from the tragus of the ear to the corner of the mouth and from the tragus of the ear to the pogonion. These measurements were taken by flexible meters product by IKEA. The data was collected in first, third, seventh days postoperatively.

\section{Post-operative trismus:}

Trismus was evaluated postoperatively at first, third, seventh days through measuring the maximum mouth opening using digital caliper applied between upper central incisors and lower central incisors at the midline. 


\section{Statistical analysis:}

Data were represented as mean and standard deviation. Repeated measures analysis of variance (ANOVA) test was used to compare numeric variables within the studied group of patients. Post Hoc test was done if ANOVA or Friedman tests were positive. Using SPSS version in all tests, result was considered statistically significant if the $p$ - value was less than 0.05 .

\section{RESULTS}

\section{Comparison between groups}

\section{1- Pain}

In the first day, a significantly higher pain score (7.942 \pm 0.812$)$ was recorded in control $(\mathrm{P}<0.0001)$, while IM and SM recorded a significantly lower mean score. Tukey's post hoc test revealed no significant difference between IM and SM groups.

In the third day, a significantly higher pain score (6.134 \pm 0.634$)$ was recorded in control $(\mathrm{P}<0.0001)$, while IM and SM recorded a significantly lower mean score. Tukey's post hoc test revealed a significant difference between each 2 groups.

In the $7^{\text {th }}$ day, a significantly higher pain score $(2.241 \pm 0.221)$ was recorded in control $(\mathrm{P}<0.0001)$, while IM and SM recorded score 0 . Tukey's post hoc test revealed no significant difference between IM and SM groups.

\section{2- Mouth opening}

Pre-operatively, a higher mean mouth opening $(40.2 \pm 1.32)$ was recorded in control, while SM recorded the lowest mean value. ANOVA and Tukey's post hoc tests revealed no significant difference between all groups.

In the 1st day, a significantly higher mean mouth opening (34.93 \pm 2.02$)$ was recorded in IM group $(\mathrm{P}<0.0001)$, followed by SM group, while control recorded a significantly lower mean score. Tukey's post hoc test revealed no significant difference between IM and SM groups.

In the third day, a significantly higher mean mouth opening (35.58 \pm 1.24$)$ was recorded in SM group $(\mathrm{P}<0.0001)$, followed by IM group, while control recorded the lowest mean score. Tukey's post hoc test revealed a significant difference between IM and control groups.

In the $7^{\text {th }}$ day, a statistically significant $(\mathrm{P}=0.001)$ higher mean mouth opening was recorded in SM group (39.6 \pm 1.44$)$ followed by IM group (39.47 \pm 1.55$)$, while control recorded the lowest mean score. Tukey's post hoc test revealed no significant difference between IM and SM groups.

\section{3 -Postoperative Edema}

In the first day, a significantly higher mean edema value (297.5 \pm 8.92$)$ was recorded in control, followed by IM, then SM group. ANOVA test and Tukey's post hoc test revealed no significant difference between all three groups.

In the third day, a significantly higher mean edema value (368.94 \pm 6.93$)$ was recorded in control, followed by IM (353.0 \pm 6.74$)$, then SM group (336.67 \pm 6.15$)$. ANOVA test and Tukey's post hoc test revealed a significant difference between all three groups $(\mathrm{P}<0.0001)$.

In the $7^{\text {th }}$ day, a significantly higher mean edema value (328.76 \pm 6.52$)$ was recorded in control, followed by IM $(312.4 \pm 6.58)$, then SM group (293.42 \pm 6.11$)$. ANOVA test and Tukey's post hoc test revealed a significant difference between all three groups $(\mathrm{P}<0.0001)$.

\section{DISCUSSION}

Dexamethasone is a highly selective, long-acting, synthetic corticosteroid, which has potent antiinflammatory action. It exerts basic glucocorticoid action and is approximately 25 times more potent than hydrocortisone, 6 times than of prednisolone, 
4 times that of methyl prednisolone and triamcinolone, and equipotent to betamethasone $\mathrm{e}^{[10,11]}$.

Effects of corticosteroids on pain control are still debated and not very clear. Studies have not been able to attribute definite analgesic properties to corticosteroids. However, thromboxane A2 levels decrease post-steroid administration, prostaglandin E2 levels, which are the main pain mediators remain unaltered $^{[12]}$.

Intramasseteric (IM) route is a relatively simple technique of administering corticosteroids to reduce exacerbated inflammatory responses. The site of injection is close to the already anesthetized region which makes it a painless procedure.

IM as well as Submucosal (SM) route also ensures immediate local availability; however, their absorption depends on the local blood flow in the area of administration and could also be influenced by the presence of infection, severe inflammation. A similar treatment strategy had already been proposed in 1975 by Messer and Keller ${ }^{[9]}$, who administered $4 \mathrm{mg}$ dexamethasone in three different parts of the masseter muscle and reported a significant reduction of pain, swelling, and trismus.

In this study, dexamethasone was injected into the masseter muscle, as described by Laureano Filho $\mathrm{J}^{(13)}$. This technique is easy and convenient for both surgeon and patient alike, as the injection site is close to the area to be operated and the injection is performed in a previously anesthetized area.

\section{CONCLUSIONS}

Dexamethasone is an effective pharmacological agent to reduce post-surgical third molar removal sequelae such as pain, swelling, and trismus.

Although steroid injection through IV route has been the traditional preferred technique owing to its faster onset of action and better pain control, dexamethasone administered through local routes such as the SM route and IM route also provides comparable control of pain and swelling and has the advantage of being injected into previously anesthetized areas and requires less technical skill and better patient compliance and comfort.

\section{REFERENCES}

1. Moraschini V, Hidalgo R, Porto Barboza Ed. Effect of submucosal injection of dexamethasone after third molar surgery: A metaanalysis of randomized controlled trials. Int J Oral Maxillofac Surg 2016; 45:232-40

2. Koçer G, Yuce E, Tuzuner Oncul A, Dereci O, Koskan O. Effect of the route of administration of methylprednisolone on oedema and trismus in impacted lower third molar surgery. Int J Oral Maxillofac Surg 2014; 43:639-43.

3. Shepherd J, Brickley M. Surgical removal of third molars. BMJ 1994; 309:620-1.

4. Sortino F, Cicciù M. Strategies used to inhibit postoperative swelling following removal of impacted lower third molar. Dent Res J (Isfahan) 2011; 8:162-71

5. Hirschmann J. Some principles of systemic glucocorticoid therapy. Clin Exp Dermatol 1986; 11:27-33.

6. Majid O, Mahmood W. Effect of submucosal and intramuscular dexamethasone on postoperative sequelae after third molar surgery: Comparative study. Br J Oral Maxillofac Surg 2011; 49:647-52.

7. Majid O. Submucosal dexamethasone injection improves quality of life measures after third molar surgery: A comparative study. J Oral Maxillofac Surg 2011; 69:2289-97.

8. Boonsiriseth K, Klongnoi B, Sirintawat N, Saengsirinavin C, Wongsirichat N. Comparative study of the effect of dexamethasone injection and consumption in lower third molar surgery. Int J Oral Maxillofac Surg 2012; 41:244-7.

9. Messer EJ, Keller JJ. The use of intraoral dexamethasone after extraction of mandibular third molars. Oral Surg Oral Med Oral Pathol 1975: 40: 594-598.

10. Grossi G, Maiorana C, Garramone R, Borgonovo A, Beretta $\mathrm{M}$, Farronato D, et al. Effect of submucosal injection of dexamethasone on postoperative discomfort after third molar surgery: A prospective study. J Oral Maxillofac Surg 2007; 65:2218-26.

11. Arakeri G, Rai KK, Shivakumar HR, Jayade B. A randomized clinical trial to compare the efficacy of submucosal aprotinin injection and intravenous dexamethasone in reducing pain and swelling after third molar surgery: A prospective study. J Maxillofac Oral Surg 2013; 12:73-9. 
12. Dionne RA, Gordon SM, Rowan J, Kent A, Brahim JS Dexamethasone suppresses peripheral prostanoid levels without analgesia in a clinical model of acute inflammation. J Oral Maxillofac Surg 2003; 61:997-1003.

13. Laureano Filho J, Maurette P, Allais M, Cotinho M, Fer- nandes C. Clinical comparative study of the effectiveness of two dosages of dexamethasone to control postoperative swelling, trismus and pain after the surgical extraction of mandibular impacted third molars. Med Oral Patol Oral Cir Bucal 2008;13: E129-32. 